\title{
Bioinformatics analysis of pathways of renal infiltrating macrophages in different renal disease models
}

\author{
Yin Celeste Cheuk ${ }^{1,2 \#}$, Pingbao Zhang ${ }^{1,2 \#}$, Shihao Xu ${ }^{1,2}$, Jiyan Wang ${ }^{1,2}$, Tian Chen ${ }^{1,2}$, Yongxin Mao ${ }^{3}$, \\ Yamei Jiang ${ }^{1,2}$, Yongsheng Luo ${ }^{1,2}$, Jingjing Guo ${ }^{1,2}$, Weixi Wang ${ }^{4}$, Ruiming Rong ${ }^{1,2}$ \\ ${ }^{1}$ Department of Urology, Zhongshan Hospital, Fudan University, Shanghai, China; ${ }^{2}$ Shanghai Key Laboratory of Organ Transplantation, Shanghai, \\ China; ${ }^{3}$ Department of Urology, Huadong Hospital, Fudan University, Shanghai, China; ${ }^{4}$ Department of Geriatrics, Zhongshan Hospital, Fudan \\ University, Shanghai, China \\ Contributions: (I) Conception and design: YC Cheuk, R Rong; (II) Administrative support: R Rong; (III) Provision of study materials or patients: All \\ authors; (IV) Collection and assembly of data: T Chen, J Wang, S Xu, Y Mao; (V) Data analysis and interpretation: Y Luo, Y Jiang, P Zhang, J Guo, \\ W Wang; (VI) Manuscript writing: All authors; (VII) Final approval of manuscript: All authors. \\ \#These authors contributed equally to the work. \\ Correspondence to: Ruiming Rong. Department of Urology, Zhongshan Hospital, Fudan University, No. 180 Fenglin Road, Shanghai 200032, China. \\ Email: rong.ruiming@zs-hospital.sh.cn.
}

Background: Recent studies have suggested that macrophages are significantly involved in different renal diseases. However, the role of these renal infiltrating macrophages has not been entirely uncovered. To further clarify the underlying mechanism and identify therapeutic targets, a bioinformatic analysis based on transcriptome profiles was performed.

Methods: Three transcription profiling datasets, GSE27045, GSE51466 and GSE75808, were obtained from the Gene Expression Omnibus (GEO). Differentially expressed genes (DEGs) were assessed by Gene Ontology (GO) functional annotation, Kyoto Encyclopedia of Genes and Genomes (KEGG) pathway analysis and gene set enrichment analysis (GSEA).

Results: The classic signaling pathways and metabolic pathways of macrophages infiltrating the kidney in different pathophysiological processes, including lupus nephritis (LN), renal crystal formation and renal ischemia-reperfusion injury (IRI), were analysed. Furthermore, the common classical pathways significantly altered in the three renal disorders were the oxidative phosphorylation, VEGF signaling and JAK/STAT signaling pathways, while the renin-angiotensin system was uniquely altered in LN, the glycolysis and gluconeogenesis pathways were uniquely altered in models of renal crystal formation, and the calcium signaling pathway was specific to renal IRI.

Conclusions: Via bioinformatics analysis, this study revealed the transcriptional features of macrophages in murine LN, renal crystal formation and IRI models, which may serve as promising targets for mechanistic research and the clinical treatment of multiple renal diseases.

Keywords: Macrophage; renal disease; bioinformatics analysis; signaling pathways

Submitted Aug 30, 2021. Accepted for publication Dec 03, 2021.

doi: $10.21037 /$ tau-21-761

View this article at: https://dx.doi.org/10.21037/tau-21-761

\footnotetext{
^ ORCID: 0000-0002-7781-3806.
} 


\section{Introduction}

Increasing evidence has shown that macrophages participate in kidney injury, repair and fibrosis in multiple experimental and human renal diseases (1). In previous research, macrophages have been categorized according to different functions: classically activated macrophages (proinflammatory M1 macrophages) and alternatively activated macrophages (profibrotic/wound healing M2 macrophages) (2). Polarization of macrophages into the M1 or M2 state can be observed in vivo. For example, Lee et al. (3) showed phenotypic switching of macrophages from the M1 to the M2 state in the repair phase of acute kidney injury (AKI), and transferring M2 macrophages induces the repair process (4). Additionally, macrophage polarization has been observed in a rhabdomyolysis-induced AKI mouse model (5). However, recent studies suggest that there is a spectrum of macrophage activation states (6). Clements et al. (7) revealed that the expression of M2-specific markers in renal $\mathrm{CD}_{11} \mathrm{~b}^{+} / \mathrm{Ly} 6 \mathrm{C}^{\text {intermediate }}$ and $\mathrm{CD} 11 \mathrm{~b}^{+} / \mathrm{Ly} 6 \mathrm{C}^{\text {low }}$ macrophage populations did not enable clear clustering of traditional M1 and M2 macrophages by ex vivo standards. Therefore, the characteristics of macrophage activation states in renal tissue should be defined based on other factors (8), such as the signaling pathways activated in the macrophages.

Systemic lupus erythematosus (SLE) is a systemic autoimmune disorder characterized by autoantibodies against self-nucleic acids and leads to inflammation and injury (9). Lupus nephritis (LN) is a major type of end-stage organ damage in SLE and is a main risk factor for mortality (10). Recent studies have reported that infiltration of macrophages is related to poor prognosis in LN (11). During active LN in nephritic NZB/W mice, renal macrophages located throughout the interstitium secrete proinflammatory cytokines and chemokines, which can also contribute to tissue repair and degradation (12). Interestingly, the phenotype of these renal macrophages is different from that of classic inflammatory macrophages (12). Therefore, immuneregulating therapies are promising for $\mathrm{LN}$ treatment. A further understanding of the renal macrophages that modulate $\mathrm{LN}$ tissue damage is needed.

The high prevalence and recurrence rate of kidney stones have been recognized as problems worldwide, and kidney stones may lead to chronic kidney disease and end-stage renal disease (13). The pathology of kidney stone formation is diverse (14). Inflammatory processes, oxidative stress, mineral and lipid metabolism, and insulin resistance are involved in calcium oxalate $(\mathrm{CaOx})$ crystal development (15).
Taguchi et al. (16) found that macrophages suppress renal crystal formation by using hyperoxaluric CSF-1-deficient mice that lack M2 macrophages. The researchers then sorted renal macrophages by flow cytometry and revealed that macrophages affect renal crystal formation during hyperoxaluria by gene array profiling. Understanding the role of macrophages in renal crystal formation can aid the treatment of renal crystal deposition.

Renal ischemia-reperfusion injury (IRI) is a leading cause of AKI and is associated with poor prognosis. A recent report revealed that macrophages play a vital role in the renal injury, repair, and fibrosis associated with AKI, but the molecular mechanism remains unclear (17). Clements et al. (7) utilized a renal bilateral IRI murine model to investigate the function of macrophages in the pathological process by sorting macrophages from the kidney for microarray analysis. Sun et al. (18) also conducted an analysis of mRNA expression profiles to define differentially expressed genes (DEGs), biological processes and pathways associated with IRI. Since understanding how macrophages are altered may provide insight into promising treatments for AKI that modulate the immune response, we aimed to characterize the molecular function of macrophages after renal IRI.

The aims of this study were to explore the role of infiltrating macrophages in different kidney diseases by analysing signaling pathways, including metabolismrelated pathways, assess the similarities and differences in macrophage function between different renal diseases, and provide therapeutic targets.

We present the following article in accordance with the STROBE reporting checklist (available at https://dx.doi. org/10.21037/tau-21-761).

\section{Methods}

\section{Data preparation}

To reveal the role of macrophages in different renal diseases, we employed expression profiling array datasets of renal macrophages. After searching the Gene Expression Omnibus (GEO) database using the keywords 'renal' and 'macrophage', we found three GEO datasets (GSE27045, GSE51466 and GSE75808) that met our criteria. The transcription profiles were obtained from the GEO (http:// www.ncbi.nlm.nih.gov/geo), a public functional genomics data repository. According to the annotation information in GEO, the probes were converted into the corresponding gene symbol. 
In the GSE27045 dataset, F4/80 macrophages were isolated from NZB/W mice in the early stage of lupus, during lupus nephritis and after induction of remission using flow cytometry. RNA was extracted and processed for hybridization on the Affymetrix GeneChip Mouse Genome 430 2.0 Array. This dataset contained six "young mac" samples (GSM667532, GSM667533, GSM667534, GSM667535, GSM667536 and GSM667537), seven "sick mac" samples (GSM667538, GSM667539, GSM667540, GSM667541, GSM667542, GSM667543, and GSM667544) and four "rem mac" samples (GSM667545, GSM667546, GSM667547, and GSM667548).

In the GSE5 1466 dataset, renal CD $11 \mathrm{~b}^{+} / \mathrm{CD} 11 \mathrm{c}^{+}$ macrophages from wild-type stone model mice and CSF1-deficient (op/op) stone model mice were sorted and investigated with the Agilent-028005 SurePrint G3 Mouse GE 8x60K Microarray. GSE51466 contained three "op/op" samples (GSM1246087, GSM1246088 and GSM1246089) and three "wild type" samples (GSM1246084, GSM1246085, and GSM1246086).

In the GSE75808 dataset, renal CD $11 b^{+} / \mathrm{Ly}_{6} \mathrm{C}^{+}$ monocyte/macrophage populations were sorted by flow cytometry at 4 h, 1 day and 9 days after AKI. Samples were processed for the Affymetrix Mouse Genome 4302.0 Array. GSE75808 contained nine "sham" samples (GSM1968231, GSM1968232, GSM1968233, GSM1968234, GSM1968235, GSM1968236, GSM1968237, GSM1968238 and GSM1968239), six "4 h IRI” samples (GSM1968225, GSM1968226, GSM1968227, GSM1968228, GSM1968229 and GSM1968230), three "24 h IRI" samples (GSM1968216, GSM1968217 and GSM1968218) and six "9-day IRI" samples (GSM1968219, GSM1968220, GSM1968221, GSM1968222, GSM1968223 and GSM1968224).

The study was conducted in accordance with the Declaration of Helsinki (as revised in 2013).

\section{Identification of DEGs}

DEGs were identified by using GEO2R (http://www.ncbi. nlm.nih.gov/geo/geo2r). GEO2R is an interactive web tool that helps users identify DEGs across experimental conditions by comparing two or more datasets in a GEO series. Probe sets without corresponding gene symbols were removed, while genes with more than one probe set were averaged. $\mid \log _{\mathrm{FC}}$ (fold change) $\mid>1$ and $\mathrm{P}$ value $<0.05$ were considered statistically significant. Principal component analysis (PCA) was performed and volcano pots were generated with online tools (https://www.omicstudio.cn/tool).

\section{GO and KEGG analyses and GSEA}

To identify functional changes in the DEGs of macrophages, biological analyses were performed using the Database for Annotation, Visualization and Integrated Discovery (DAVID; http://david.ncifcrf.gov) online database. Analyses were performed via the Gene Ontology (GO) and Kyoto Encyclopedia of Genes and Genomes (KEGG) databases. A Benjamini-Hochberg corrected $P$ value $<0.05$ was considered significant. The GO and KEGG analyses were performed under $\mathrm{R}$ version 3.6.1. Gene set enrichment analysis (GSEA) was performed via online tools (https://www.omicstudio.cn/ tool). A P value $<0.05$ was considered statistically significant.

\section{Statistical analysis}

We used GEO2R to identify DEGs, and the thresholds were set as $\left|\log _{\mathrm{FC}}\right|>1$ and $\mathrm{P}<0.05$. $\mathrm{P}<0.05$ was considered statistically significant for the GO and KEGG analyses and GSEA.

\section{Results}

\section{The role of macrophages in murine systemic lupus erythematosus nephritis}

GSE27045 had three groups: a group of renal isolated macrophages taken at an early stage of lupus (the young mac group), a group of renal isolated macrophages taken during lupus nephritis (the sick mac group) and a group of renal isolated macrophages taken after induction of remission (the rem mac group). Six young mac samples, seven sick mac samples and four rem mac samples were assessed in the current study. PCA was used to compare the transcription profiles between the young mac group, sick mac group and rem group. The plot showed a clear distinction among the three groups (Figure 1A). Volcano plots were generated to visualize the distribution of expressed genes $(\mathrm{P}<0.05$ and $\left|\log _{\mathrm{FC}}\right|>1$, Figure $\left.1 B\right)$ between the young mac group and sick mac group and between the sick mac group and rem group. Compared to the young mac group, the sick mac group showed altered expression of functional genes, which were annotated based on GO biological process terms (Figure 1C). The results indicated that the upregulated genes were enriched in immune system process, the innate immune response, the inflammatory response, the adaptive immune response, the defense response to bacterium, phagocytosis recognition, the cellular response to interleukin-1, phagocytosis, engulfment, the immune 
A

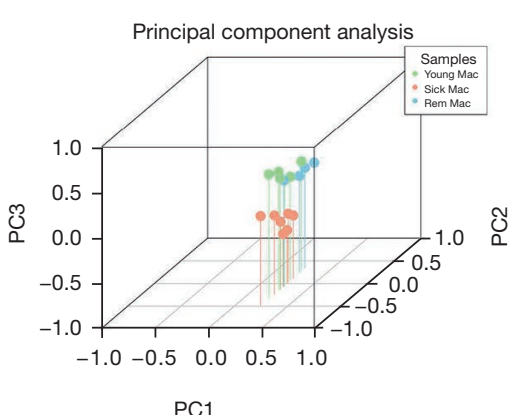

C

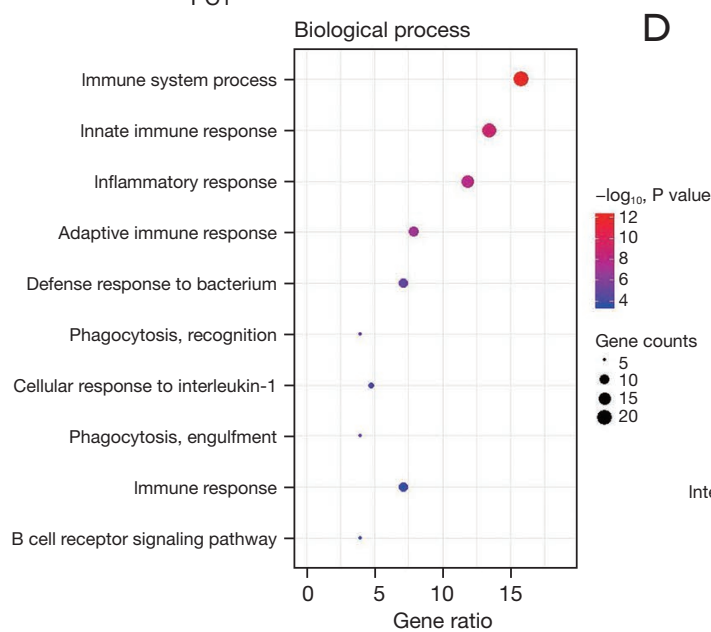

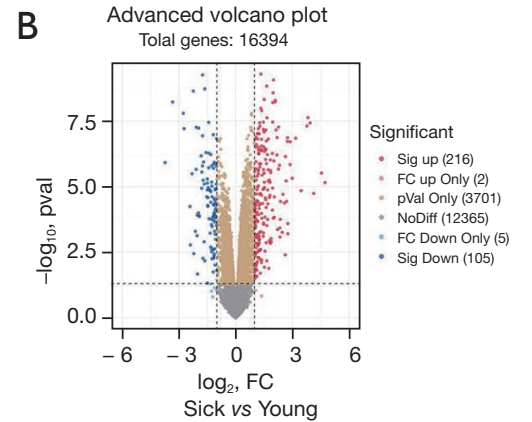
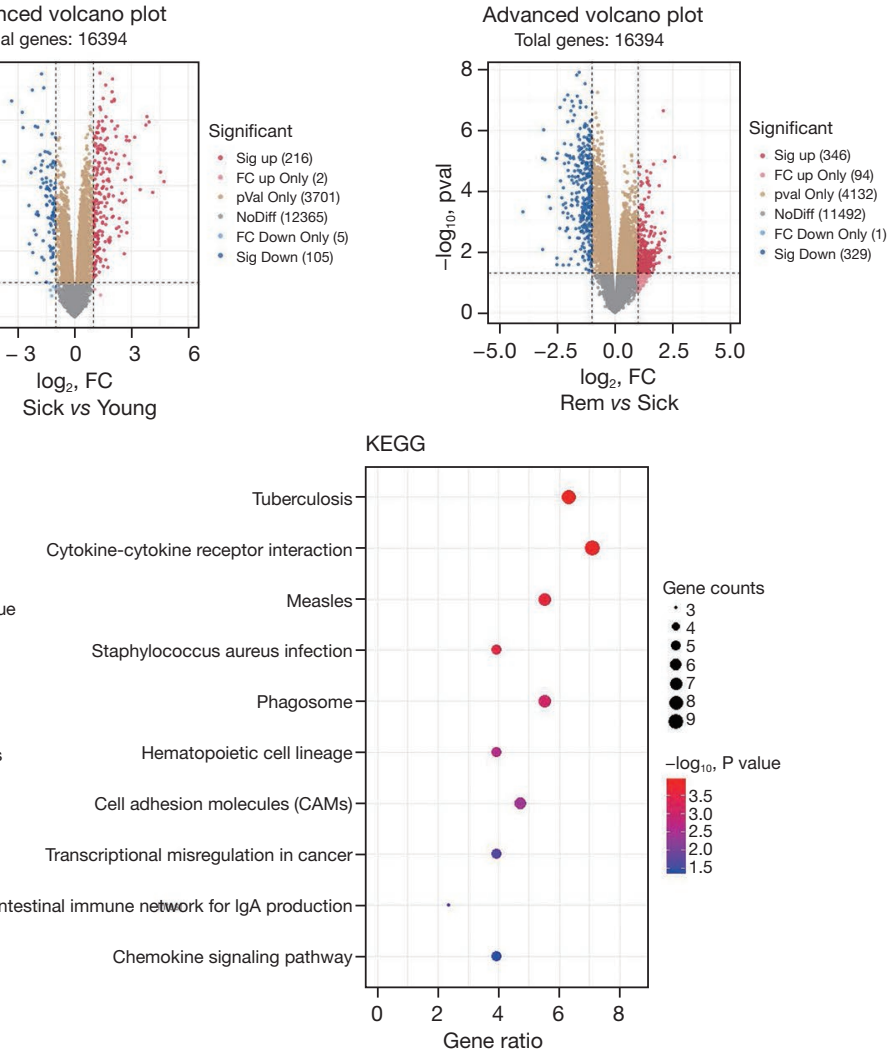

$\mathrm{E}$
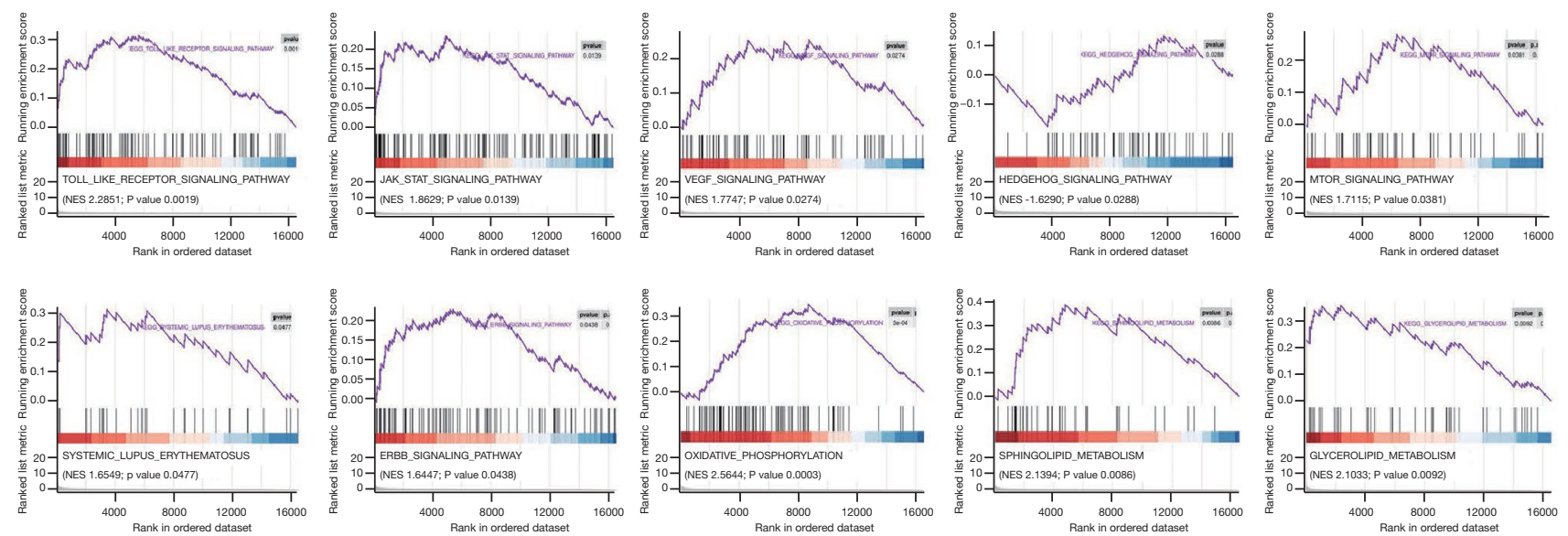

Figure 1 The characteristic and pathways of macrophages involved in murine systemic lupus erythematosus nephritis. (A) Principal component analysis (PCA) plot of the separation between the group of samples taken in the early-stage lupus (the young mac group), the group of samples taken during lupus nephritis (the sick mac group) and the group of samples taken after induction of remission (the rem mac group); (B) volcano plot of differentially expressed genes (DEGs) between the young mac group and sick mac group and between the sick mac group and rem group $\left(\mathrm{P}<0.05\right.$ and $\left.\left|\log _{\mathrm{FC}}\right|>1\right)$; (C) the enriched biological process terms of the DEGs as identified via Gene Ontology (GO) functional annotation; (D) the enriched Kyoto Encyclopedia of Genes and Genomes (KEGG) pathway terms of the DEGs; (E) gene set enrichment analysis (GSEA) was performed to identify significantly enriched pathways. 
response and the B cell receptor signaling pathway. Altered signaling pathways were also demonstrated in the KEGG pathway analysis (Figure 1D), including pathways related to tuberculosis, cytokine-cytokine receptor interactions, measles, Staphylococcus aureus infection, phagosomes, the haematopoietic cell lineage, cell adhesion molecules (CAMs), transcriptional misregulation in cancer, the intestinal immune network for IgA production and the chemokine signaling pathway. As we wanted to focus on classical signaling pathways and metabolic processes, GSEA was applied to reveal the crucial signaling pathways and metabolism-related pathways of macrophages in SLE nephritis (Figure 1E); the results included the terms Toll-like receptor signaling pathway [Normalized Enrichment Score $(\mathrm{NES})=2.2851 ; \mathrm{P}=0.0019]$, JAK-STAT signaling pathway (NES $=1.8629 ; \mathrm{P}=0.0139), \mathrm{VEGF}$ signaling pathway (NES $=1.7747 ; \mathrm{P}=0.0274$ ), Hedgehog signaling pathway $(\mathrm{NES}=-1.6290 ; \mathrm{P}=0.0288)$, MTOR signaling pathway (NES $=1.7115 ; \mathrm{P}=0.0381$ ), systemic lupus erythematosus $(\mathrm{NES}=1.6549 ; \mathrm{P}=0.0477)$, ERBB signaling pathway (NES $=1.6447 ; \mathrm{P}=0.0438$ ), oxidative phosphorylation (NES =2.5644; $\mathrm{P}=0.0003$ ), sphingolipid metabolism (NES $=2.1394 ; \mathrm{P}=0.0086)$ and glycerolipid metabolism (NES $=2.1033 ; \mathrm{P}=0.0092)$.

\section{The role of macrophages in murine renal crystal formation}

GSE51466 had two groups, including a group of op/op (CSF-1-deficient) renal macrophage that received glyoxylate (GOX) injection (op/op group) for 6 days and a group of wild-type renal macrophage that received GOX injection for 6 days (wild-type group). Three op/op samples and three wild-type samples were assessed in the present study. The PCA results showed a clear distinction between the samples (Figure $2 A$ ). As shown in the volcano plots in Figure $2 B$, upregulated and downregulated DEGs $\left(\mathrm{P}<0.05\right.$ and $\left.\left|\log _{\mathrm{FC}}\right|>1\right)$ were identified in the dataset. To obtain deeper insights into the biological function of the DEGs, we analysed the GO terms. The DEGs were most significantly enriched in biological process terms such as immune response, inflammatory response, chemokinemediated signaling pathway, positive regulation of smooth muscle cell proliferation, chemotaxis, cellular response to lipopolysaccharide, response to molecule of bacterial origin, cell chemotaxis, positive regulation of NF-kappaB import into nucleus, and cellular response to interferon-gamma (Figure 2C). KEGG pathway analysis was performed to investigate the involved signaling pathways, and the results included pathway related to the cytokine-cytokine receptor interaction, rheumatoid arthritis, graft-versus-host disease, the NOD-like receptor signaling pathway, the TNF signaling pathway, influenza $\mathrm{A}$, antigen processing and presentation, Staphylococcus aureus infection, complement and coagulation cascades, and type I diabetes mellitus (Figure 2D). Furthermore, by using GSEA (Figure 2E), we found enrichment of the ERBB signaling pathway (NES $=1.8573 ; \mathrm{P}=0.0001$ ), JAK-STAT signaling pathway (NES $=1.5519 ; \mathrm{P}=0.0002)$, MAPK signaling pathway (NES $=1.5043 ; \mathrm{P}=0.0001$ ), MTOR signaling pathway (NES $=1.9725 ; \mathrm{P}=0.0001)$, PPAR signaling pathway $(\mathrm{NES}=1.5758$; $\mathrm{P}=0.0025)$, Toll-like receptor signaling pathway (NES $=1.9680 ; \mathrm{P}=0.0001$ ), and VEGF signaling pathway (NES $=1.7056 ; \mathrm{P}=0.0002$ ), which are all well-known pathways related to the suppressive role of macrophages in renal crystal formation. Additionally, other enriched pathways suggested the involvement of macrophages in metabolismrelated mechanisms, such as oxidative phosphorylation (NES $=1.9923 ; \mathrm{P}=0.0001)$, purine metabolism $(\mathrm{NES}=1.5497$; $\mathrm{P}=0.0001$ ), fatty acid metabolism (NES $=1.7238 ; \mathrm{P}=0.0023$ ), fructose and mannose metabolism $(\mathrm{NES}=1.8738 ; \mathrm{P}=0.0012$ ) and pyrimidine metabolism (NES $=1.8466 ; \mathrm{P}=0.0001$ ).

\section{The role of macrophages after murine renal ischemia-reperfusion injury}

GSE75808 had four groups, including a group that received sham surgery (sham group), a group with samples taken $4 \mathrm{~h}$ postperfusion after IRI (4 h group), a group with samples taken $24 \mathrm{~h}$ postperfusion after IRI (1-day group) and a group of samples taken 9 days postperfusion after IRI (9-day group). Nine sham samples, six 4-h samples, three 1-day samples and six 9-day samples were assessed in the present study. The PCA plot showed separation between the sham, $4 \mathrm{~h}$ after IRI, 1-day and 9-day samples (Figure $3 A$ ). These results were also displayed visually in the form of a volcano plot $\left(\mathrm{P}<0.05\right.$ and $\left|\log _{\mathrm{FC}}\right|>1$, Figure $\left.3 B\right)$. The BP terms (Figure $3 C$ ) enriched by the DEGs were mainly associated with RNA splicing, protein transport, mRNA processing, 2-oxoglutarate metabolic processes, transport, ubiquinone biosynthetic processes, the response to stress, oxidationreduction processes, the tricarboxylic acid cycle, and isocitrate metabolic processes. Moreover, KEGG pathway analysis (Figure 3D) illustrated significant enrichment of pathways related to the biosynthesis of antibiotics, carbon metabolism, the citrate cycle (TCA cycle), glutathione metabolism, metabolic pathways, the TNF signaling 
A
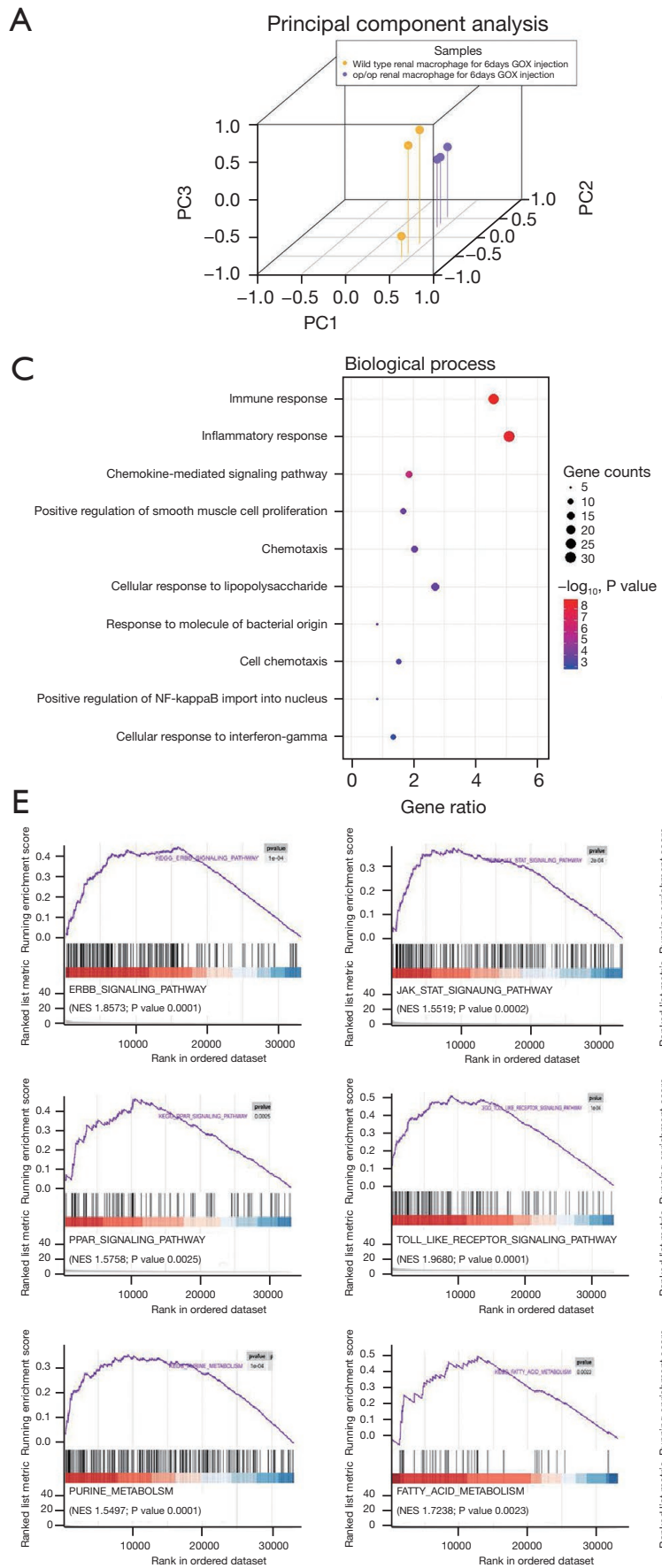

Gene ratio
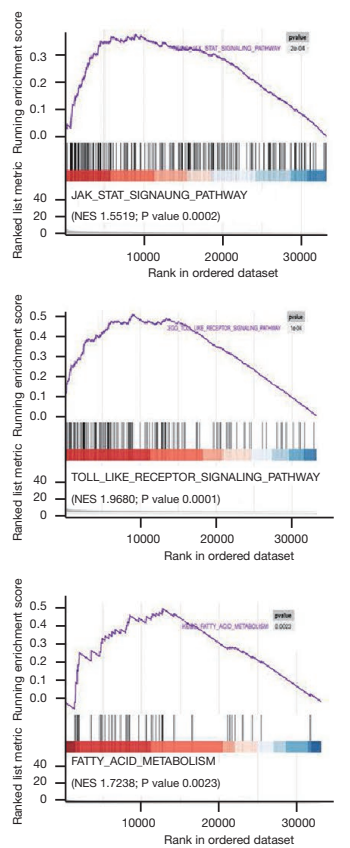
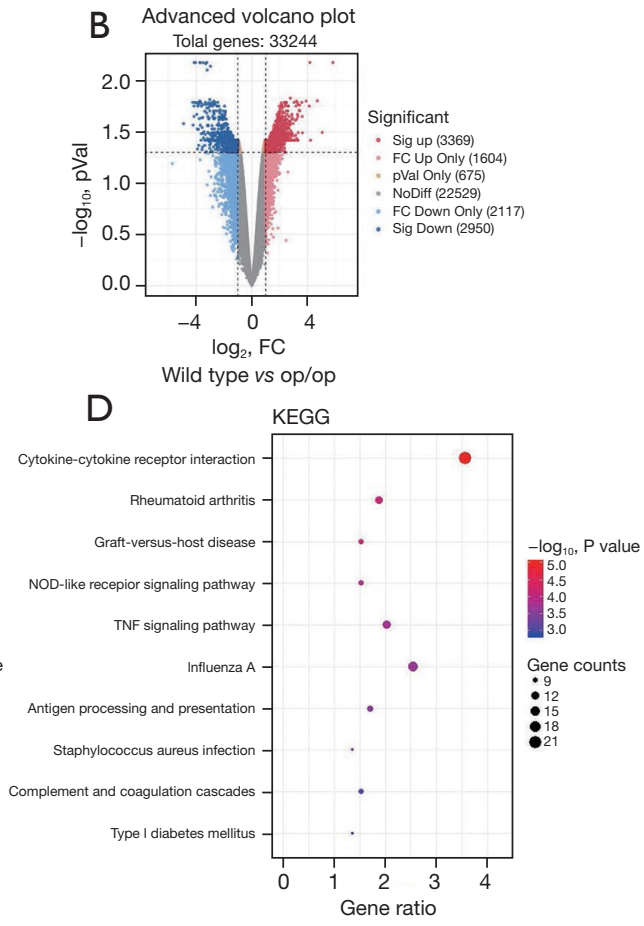
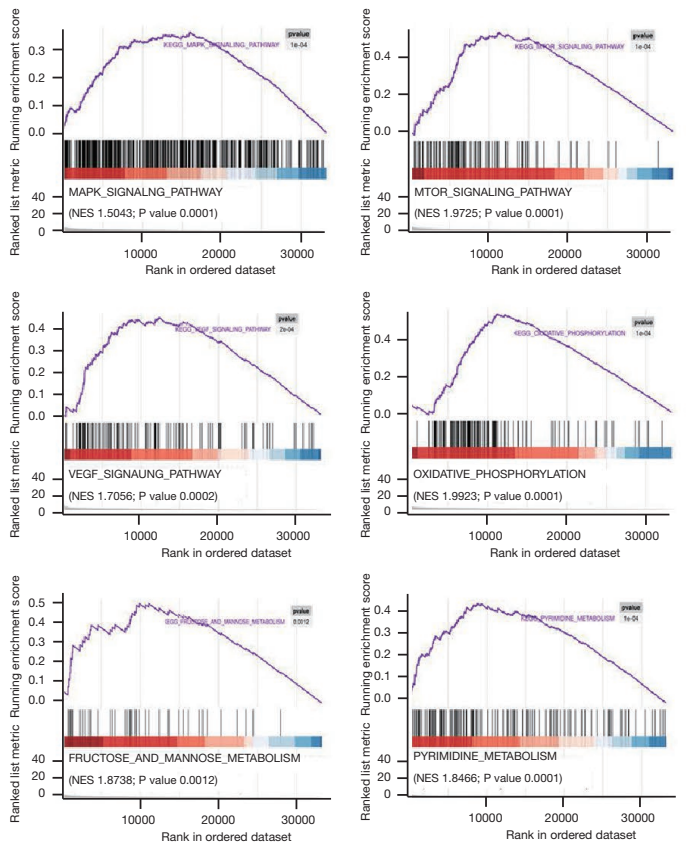

Figure 2 The characteristic and pathways of macrophages involved in murine renal crystal formation. (A) Principal component analysis (PCA) plot of the separation between the group of wild-type renal macrophages treated with GOX injection for 6 days (the wild-type group) and the group of op/op (CSF-1-deficient) renal macrophage treated with glyoxylate (GOX) injection for 6 days (the op/op group); (B) volcano plot of the differentially expressed genes (DEGs) between the wild-type group and the op/op group $\left(\mathrm{P}<0.05\right.$ and $\left.\left|\log _{\mathrm{FC}}\right|>1\right)$; (C) the enriched biological process terms of the DEGs as identified via Gene Ontology (GO) functional annotation; (D) the enriched Kyoto Encyclopedia of Genes and Genomes (KEGG) pathway terms of the DEGs; (E) gene set enrichment analysis (GSEA) was performed to identify significantly enriched pathways. 
A

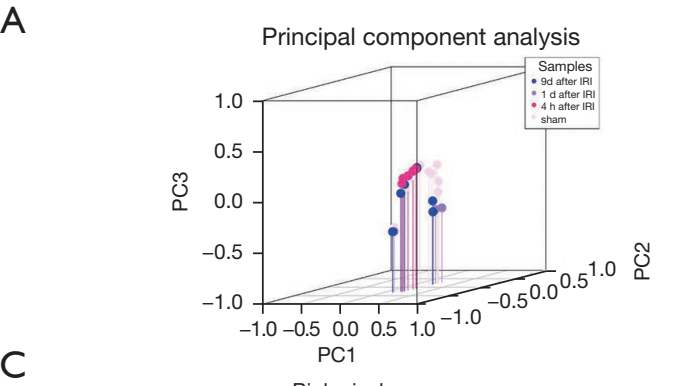

C
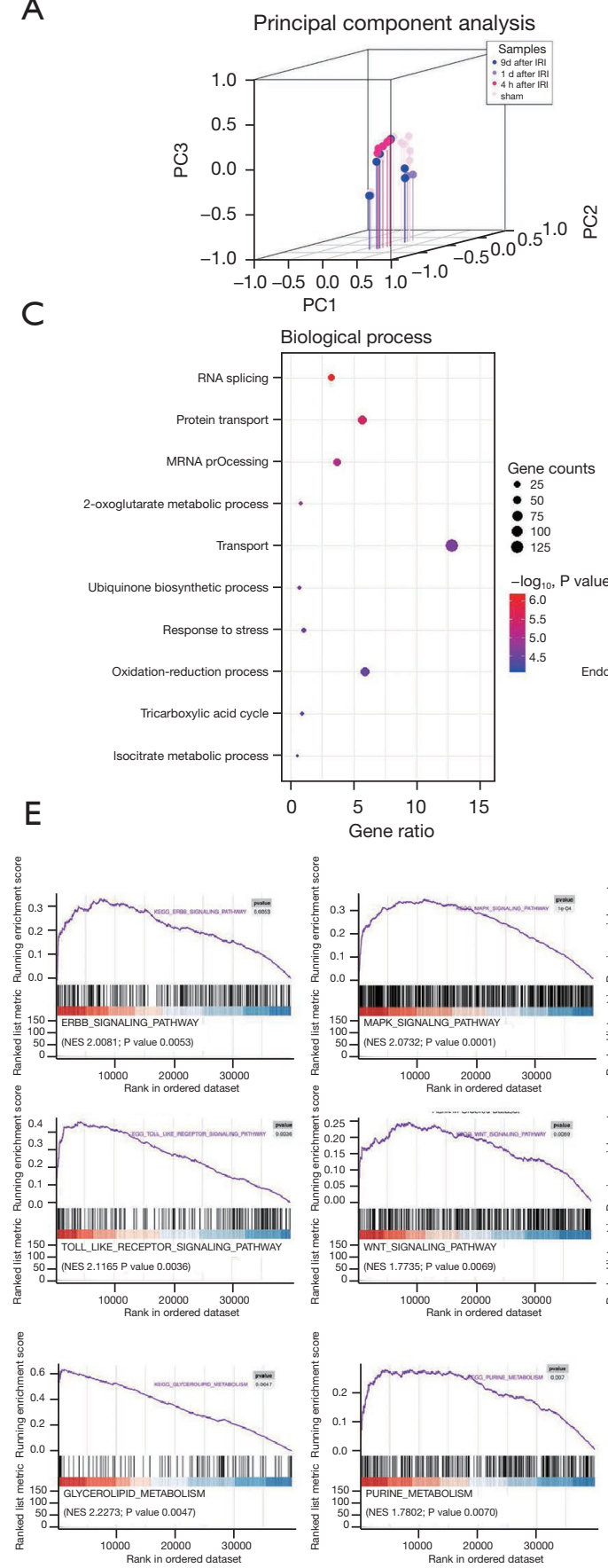

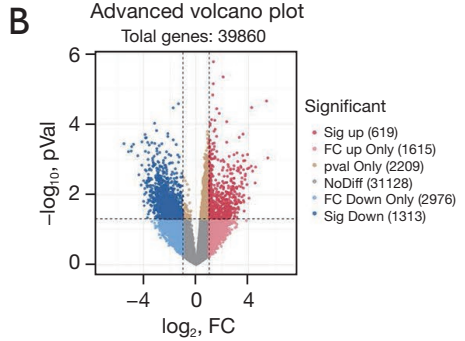

D
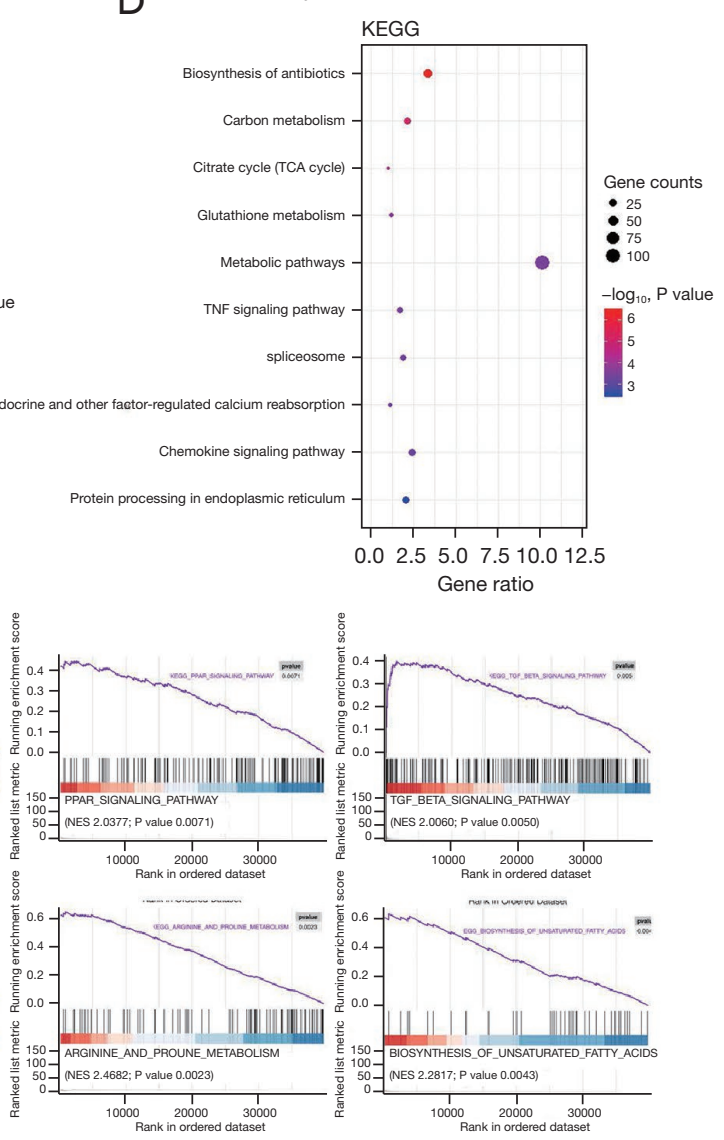

$\mathrm{F}$

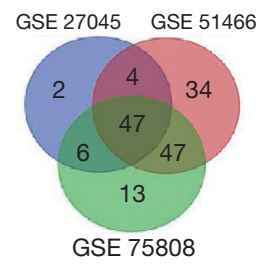

Figure 3 The characteristic and pathways of macrophages involved in murine renal ischemia-reperfusion injury (IRI). (A) Principal component analysis (PCA) plot of the separation between the sham surgery (sham) group, the group of samples taken $4 \mathrm{~h}$ postperfusion after IRI (the $4 \mathrm{~h}$ group), the group of samples taken $24 \mathrm{~h}$ postperfusion after IRI (the 1-day group) and the group of samples taken 9 days postperfusion after IRI (the 9-day group); (B) volcano plot of the differentially expressed genes (DEGs) between the 1-day group and sham group $\left(\mathrm{P}<0.05\right.$ and $\left.\mid \log _{\mathrm{FC}} \mathrm{I}>1\right)$; (C) the enriched biological process terms of the DEGs as identified via Gene Ontology (GO) functional annotation; (D) the enriched Kyoto Encyclopedia of Genes and Genomes (KEGG) pathway terms of the DEGs; (E) gene set enrichment analysis (GSEA) was performed to identify significantly enriched pathways; (F) the Venn diagram represents common and unique pathways that were significantly altered in different datasets. 
pathway, the spliceosome, endocrine- and other factorregulated calcium reabsorption, chemokine signaling pathways and protein processing in the endoplasmic reticulum. The significantly enriched KEGG pathways in 1-day $v s$. sham samples identified in the GSEA are displayed in Figure $3 E$ and mainly included the ERBB signaling pathway (NES $=2.0081 ; \mathrm{P}=0.0053)$, MAPK signaling pathway (NES $=2.0732 ; \mathrm{P}=0.0001$ ), PPAR signaling pathway $(\mathrm{NES}=2.0377 ; \mathrm{P}=0.0071)$, TGF $\beta$ signaling pathway (NES $=2.0060 ; \mathrm{P}=0.0050)$, Toll-like receptor signaling pathway (NES $=2.1165 ; \mathrm{P}=0.0036$ ), WNT signaling pathway (NES $=1.7735 ; \mathrm{P}=0.0069)$, pathways related to arginine and proline metabolism (NES $=2.4682 ; \mathrm{P}=0.0023$ ), pathways related to the biosynthesis of unsaturated fatty acids (NES $=2.2817 ; \mathrm{P}=0.0043)$, pathways related to glycerolipid metabolism (NES $=2.2273 ; \mathrm{P}=0.0047)$ and pathways related to purine metabolism (NES $=1.7802 ; \mathrm{P}=0.0070)$.

Furthermore, a Venn diagram (Figure $3 F$ ) of the common and unique pathways that were significantly altered in different diseases was generated. Among the 47 pathways identified from all the datasets, the oxidative phosphorylation pathway, VEGF signaling pathway and JAK/STAT signaling pathway were common to all datasets. Of the two pathways unique to the GSE27045 dataset, the renin-angiotensin system pathway is a classical pathway and was thus considered worthy of further study. Of the 34 pathways specific to the GSE51466 dataset, the glycolysis and gluconeogenesis pathways are well known. Of the 13 pathways specific to the GSE75808 dataset, the calcium signaling pathway is a classical pathway.

\section{Discussion}

Increasing evidence shows that multiple signals are involved in macrophage activation in various diseases (2), such as signals from the JAK/STAT, PI3K/AKT, NF-кB, JNK, Wnt/ $\beta$-catenin and Notch signaling pathways (19-23). Interestingly, strategies inducing metabolic reprogramming have great potential for regulating macrophages and in the development of novel approaches. Metabolic pathways that modulate the activation of macrophages include the glycolysis, TCA cycle, and fatty acid metabolism pathways, while metabolites such as succinate, $\alpha$-ketoglutarate, itaconate, amino acids, adenosine and lactate also drive macrophage activation (24-26). In this regard, therapeutic strategies targeting signaling pathways and metabolic processes involved in the recruitment and activation of macrophages deserve attention for the treatment of kidney disease in the future. In the current study, we investigated the role of infiltrating macrophages in different renal diseases (including LN, renal crystal formation and IRI) by analysing classic signaling pathways and metabolic pathways. Three GEO datasets (GSE27045, GSE51466 and GSE75808) were included in our study.

In GSE27045, Bethunaickan et al. (12) showed that the traditional inflammatory macrophage population found in most acute renal inflammatory models was decreased in NZB/W mice and other murine SLE models. In our study, macrophages in LN were found to be mainly involved in pathways such as the ERBB signaling pathway, Hedgehog signaling pathway, JAK-STAT signaling pathway, MTOR signaling pathway, Toll-like receptor signaling pathway and VEGF signaling pathway. Furthermore, glycerolipid metabolism, oxidative phosphorylation and sphingolipid metabolism were found to be related to macrophages in LN. Moreover, compared with the pathways enriched in macrophages in renal crystal formation and IRI, the reninangiotensin system pathway was found to be uniquely enriched in macrophages in LN. The renin-angiotensin system may influence immune responses and particularly affect macrophages through different signaling pathways. Hammer et al. (27) identified that the Ang-[1-7]/Mas axis is an important intracellular signaling pathway for macrophage function in vascular system and the central nervous system inflammation. The renin-angiotensin system impairs macrophage lipid metabolism in age-related macular degeneration in mouse models (28). The renin-angiotensin system also plays a role in LN. Yue et al. (29) revealed that blocking the renin-angiotensin system early improves renal outcomes of SLE patients with antiphospholipid-associated nephropathy. In a murine model, Matsuki-Muramoto et al. (30) verified that bortezomib treatment prevents glomerulosclerosis related to $\mathrm{LN}$ by suppressing the immune and renin-angiotensin systems. Thus, identifying enrichment of these key pathways, particularly the enrichment of the renin-angiotensin system, may provide new targets for modulating macrophages to further control the $\mathrm{LN}$ process.

In the gene array profiling of GSE51466, Taguchi et al. (16) compared crystal formation and macrophage polarization in renal tissue between wild-type and CSF1-deficient mice after inducing hyperoxaluria. The results indicated a suppressive function of macrophages in renal crystal formation. Moreover, we demonstrated that the differentially altered mRNAs between macrophages taken from the kidneys of wild-type or CSF-1-deficient mice 
were mainly involved in the ERBB signaling pathway, JAK-STAT signaling pathway, MAPK signaling pathway, MTOR signaling pathway, PPAR signaling pathway, Tolllike receptor signaling pathway, and VEGF signaling pathway. In addition, the oxidative phosphorylation, purine metabolism, fatty acid metabolism, fructose and mannose metabolism and pyrimidine metabolism pathways were identified as enriched metabolic pathways that may participate in the suppressive role of macrophages in renal crystal formation. Furthermore, compared with the pathways associated with macrophages in LN and IRI, glycolysis and gluconeogenesis were found to be activated in macrophages during renal crystal formation. According to stimuli from the microenvironment, macrophages can switch from aerobic oxidative phosphorylation to high rates of anaerobic glycolysis (31), and proinflammatory macrophages utilize glycolysis and the pentose phosphate pathway to meet their ATP requirements (32). The role of glycolysis in macrophages during renal crystal formation still needs further study.

With the GSE75808 dataset, Sun et al. (18) revealed that the proportion of macrophages peaked one day after IRI and was significantly decreased at Day 9 after IRI. Thus, we chose the macrophage profile data from one day after IRI to compare with those of the sham group. Studies have also shown that Atp5a1, Atp5o, Cdc42, Cox4i, Nhp2 and Rac2 are critical genes involved in oxidation-reduction, migration, apoptosis, and macrophage proliferation and polarization (18). We focused on specific signaling pathways to explore novel targets that can influence AKI. Compared to the sham group, the group with macrophages taken from the kidney 1 day after IRI showed significant enrichment of pathways including the ERBB signaling pathway, MAPK signaling pathway, PPAR signaling pathway, TGF $\beta$ signaling pathway, Toll-like receptor signaling pathway and WNT signaling pathway. Regarding metabolic pathways, the arginine and proline metabolism pathways, pathways related to the biosynthesis of unsaturated fatty acids, the glycerolipid metabolism pathway and the purine metabolism pathway were identified. Interestingly, compared with the pathways enriched in macrophages in LN and renal crystal formation, the calcium signaling pathway was found to be specifically enriched in macrophages in IRI. Calcium has been found to be central to various physiological processes, such as immune system activation and maintenance (33). Intracellular $\mathrm{Ca}^{2+}$ fluctuations act on many cellular functions in monocytes/macrophages, including chemotaxis and phagocytosis (34). $\mathrm{Ca}^{2+}$ also plays crucial roles in actin rearrangement and the activation of $\mathrm{NADPH}$ oxidase and protein kinase $\mathrm{C}$ in phagocytic cells like macrophages (35). In a mouse model of AKI, Wasnik et al. (36) demonstrated that blocking intracellular calcium improved survival. In primary tubular epithelial cells, TRPC6-mediated $\mathrm{Ca}^{2+}$ influx was found by Hou et al. (37) to suppress cytoprotective autophagy triggered by oxidative stress by downregulating PI3K/AKT and ERK signaling, increasing the autophagy level, and restraining the apoptosis mediated by mitochondria. These studies and results suggest that understanding the abovementioned signaling pathways, especially the calcium signaling pathway, in macrophages during IRI may provide new targets for treatment.

In summary, our study identified transcriptional features of macrophages in murine $\mathrm{LN}$, renal crystal formation and IRI models. We focused on the classic signaling pathways and metabolic pathways of macrophages infiltrating the kidney during these pathophysiological processes. These results help uncover the comprehensive role of macrophages in multiple renal diseases at the transcript level, which could provide vital information for reversing different renal diseases. However, there are some limitations of this study. First, the identified signaling pathways require verification for further applications. Second, the abilities of macrophages in various renal diseases need to be further validated.

\section{Conclusions}

In this investigation, our data based on transcriptional profiles of macrophages provide a deeper understanding of the classic signaling pathways and metabolic pathways activated in renal infiltrating macrophages in LN, renal crystal formation and IRI models. We also explored the similarities and differences in macrophage function among these different renal diseases. Oxidative phosphorylation, the VEGF signaling pathway, and the JAK/STAT signaling pathway were found to be activated in macrophages in all the three renal diseases; the renin-angiotensin system was found to be activated in macrophages in LN; glycolysis and gluconeogenesis were found to be activated in macrophages during renal crystal formation; and the calcium signaling pathway was found to be activated in macrophages in renal IRI. These specific pathways deserve further study and may represent promising therapeutic targets.

\section{Acknowledgments}

Funding: This study was supported by the National Key 
R\&D Program of China (2018YFA0107501) and National Natural Science Foundation of China (81770747 and 81970646).

\section{Footnote}

Reporting Checklist: The authors have completed the STROBE reporting checklist. Available at https://dx.doi. org/10.21037/tau-21-761

Peer Review File: Available at https://dx.doi.org/10.21037/ tau-21-761

Conflicts of Interest: All authors have completed the ICMJE uniform disclosure form (available at https://dx.doi. org/10.21037/tau-21-761). The authors have no conflicts of interest to declare.

Ethical Statement: The authors are accountable for all aspects of the work in ensuring that questions related to the accuracy or integrity of any part of the work are appropriately investigated and resolved. The study was conducted in accordance with the Declaration of Helsinki (as revised in 2013).

Open Access Statement: This is an Open Access article distributed in accordance with the Creative Commons Attribution-NonCommercial-NoDerivs 4.0 International License (CC BY-NC-ND 4.0), which permits the noncommercial replication and distribution of the article with the strict proviso that no changes or edits are made and the original work is properly cited (including links to both the formal publication through the relevant DOI and the license). See: https://creativecommons.org/licenses/by-nc-nd/4.0/.

\section{References}

1. He YX, Diao TT, Song SM, et al. Wnt4 is significantly upregulated during the early phases of cisplatin-induced acute kidney injury. Sci Rep 2018;8:10555.

2. Meng XM, Mak TS, Lan HY. Macrophages in Renal Fibrosis. Adv Exp Med Biol 2019;1165:285-303.

3. Lee S, Huen S, Nishio H, et al. Distinct macrophage phenotypes contribute to kidney injury and repair. J Am Soc Nephrol 2011;22:317-26.

4. Xie X, Yang X, Wu J, et al. Trib1 Contributes to Recovery From Ischemia/Reperfusion-Induced Acute Kidney Injury by Regulating the Polarization of Renal Macrophages.
Front Immunol 2020;11:473.

5. Belliere J, Casemayou A, Ducasse L, et al. Specific macrophage subtypes influence the progression of rhabdomyolysis-induced kidney injury. J Am Soc Nephrol 2015;26:1363-77.

6. Feketea G, Bocsan CI, Popescu C, et al. A Review of Macrophage MicroRNAs' Role in Human Asthma. Cells 2019;8:420.

7. Clements M, Gershenovich $M$, Chaber C, et al. Differential Ly6C Expression after Renal IschemiaReperfusion Identifies Unique Macrophage Populations. J Am Soc Nephrol 2016;27:159-70.

8. Murray PJ, Allen JE, Biswas SK, et al. Macrophage activation and polarization: nomenclature and experimental guidelines. Immunity 2014;41:14-20.

9. Zhou X, Chen H, Wei F, et al. 3beta-Acetyloxy-oleanolic Acid Attenuates Pristane-Induced Lupus Nephritis by Regulating Th17 Differentiation. J Immunol Res 2019;2019:2431617.

10. Berthier CC, Bethunaickan R, Gonzalez-Rivera T, et al. Cross-species transcriptional network analysis defines shared inflammatory responses in murine and human lupus nephritis. J Immunol 2012;189:988-1001.

11. Maria NI, Davidson A. Renal Macrophages and Dendritic Cells in SLE Nephritis. Curr Rheumatol Rep 2017;19:81.

12. Bethunaickan R, Berthier CC, Ramanujam M, et al. A unique hybrid renal mononuclear phagocyte activation phenotype in murine systemic lupus erythematosus nephritis. J Immunol 2011;186:4994-5003.

13. Taguchi K, Okada A, Hamamoto S, et al. M1/M2macrophage phenotypes regulate renal calcium oxalate crystal development. Sci Rep 2016;6:35167.

14. Manzoor MAP, Agrawal AK, Singh B, et al. Morphological characteristics and microstructure of kidney stones using synchrotron radiation muCT reveal the mechanism of crystal growth and aggregation in mixed stones. PLoS One 2019;14:e214003.

15. Taguchi K, Okada A, Unno R, et al. Macrophage Function in Calcium Oxalate Kidney Stone Formation: A Systematic Review of Literature. Front Immunol 2021;12:673690.

16. Taguchi K, Okada A, Kitamura H, et al. Colonystimulating factor-1 signaling suppresses renal crystal formation. J Am Soc Nephrol 2014;25:1680-97.

17. Yang Q, Wang Y, Pei G, et al. Bone marrow-derived Ly6C- macrophages promote ischemia-induced chronic kidney disease. Cell Death Dis 2019;10:291.

18. Sun D, Wan X, Pan BB, et al. Bioinformatics Analysis of Genes and Pathways of CD11b+/Ly6Cintermediate 
Macrophages after Renal Ischemia-Reperfusion Injury. Curr Med Sci 2018;38:70-7.

19. Chen W, Liu Y, Chen J, et al. The Notch signaling pathway regulates macrophage polarization in liver diseases. Int Immunopharmacol 2021;99:107938.

20. Kwon YJ, Seo EB, Kim SK, et al. Chamaecyparis obtusa (Siebold \& Zucc.) Endl. leaf extracts prevent inflammatory responses via inhibition of the JAK/STAT axis in RAW264.7 cells. J Ethnopharmacol 2022;282:114493.

21. Le F, Yang L, Han Y, et al. TPL Inhibits the Invasion and Migration of Drug-Resistant Ovarian Cancer by Targeting the PI3K/AKT/NF-kappaB-Signaling Pathway to Inhibit the Polarization of M2 TAMs. Front Oncol 2021;11:704001.

22. Zhang W, Fu Z, Yin H, et al. Macrophage Polarization Modulated by Porcine Circovirus Type 2 Facilitates Bacterial Coinfection. Front Immunol 202 1;12:688294.

23. Yang Y, Ye YC, Chen Y, et al. Crosstalk between hepatic tumor cells and macrophages via $\mathrm{Wnt} /$ beta-catenin signaling promotes M2-like macrophage polarization and reinforces tumor malignant behaviors. Cell Death Dis 2018;9:793.

24. Mehla K, Singh PK. Metabolic Regulation of Macrophage Polarization in Cancer. Trends Cancer 2019;5:822-34.

25. Wilson JL, Mayr HK, Weichhart T. Metabolic Programming of Macrophages: Implications in the Pathogenesis of Granulomatous Disease. Front Immunol 2019;10:2265.

26. Batista-Gonzalez A, Vidal R, Criollo A, et al. New Insights on the Role of Lipid Metabolism in the Metabolic Reprogramming of Macrophages. Front Immunol 2020;10:2993.

27. Hammer A, Stegbauer J, Linker RA. Macrophages in neuroinflammation: role of the renin-angiotensin-system. Pflugers Arch 2017;469:431-44.

28. Nagai N, Kawashima H, Toda E, et al. Renin-angiotensin

Cite this article as: Cheuk YC, Zhang P, Xu S, Wang J, Chen T, Mao Y, Jiang Y, Luo Y, Guo J, Wang W, Rong R. Bioinformatics analysis of pathways of renal infiltrating macrophages in different renal disease models. Transl Androl Urol 2021;10(12):4333-4343. doi: 10.21037/tau-21-761 system impairs macrophage lipid metabolism to promote age-related macular degeneration in mouse models. Commun Biol 2020;3:767.

29. Yue C, Li G, Wen Y, et al. Early Renin-angiotensin System Blockade Improved Short-term and Longterm Renal Outcomes in Systemic Lupus Erythematosus Patients with Antiphospholipid-associated Nephropathy. J Rheumatol 2018;45:655-62.

30. Matsuki-Muramoto Y, Nozawa K, Uomori K, et al. Bortezomib treatment prevents glomerulosclerosis associated with lupus nephritis in a murine model through suppressive effects on the immune and renin-angiotensin systems. Mod Rheumatol 2017;27:77-86.

31. Viola A, Munari F, Sánchez-Rodríguez R, et al. The Metabolic Signature of Macrophage Responses. Front Immunol 2019;10:1462.

32. Artyomov MN, Sergushichev A, Schilling JD. Integrating immunometabolism and macrophage diversity. Semin Immunol 2016;28:417-24.

33. Froghi S, Grant CR, Tandon R, et al. New Insights on the Role of TRP Channels in Calcium Signaling and Immunomodulation: Review of Pathways and Implications for Clinical Practice. Clin Rev Allergy Immunol 2021;60:271-92.

34. Feske S, Wulff H, Skolnik EY. Ion channels in innate and adaptive immunity. Annu Rev Immunol 2015;33:291-353.

35. Pradhan G, Raj Abraham P, Shrivastava R, et al. Calcium Signaling Commands Phagosome Maturation Process. Int Rev Immunol 2019;38:57-69.

36. Wasnik S, Tang X, Bi H, et al. IGF-1 Deficiency Rescue and Intracellular Calcium Blockade Improves Survival and Corresponding Mechanisms in a Mouse Model of Acute Kidney Injury. Int J Mol Sci 2020;21:4095.

37. Hou X, Huang M, Zeng X, et al. The Role of TRPC6 in Renal Ischemia/Reperfusion and Cellular Hypoxia/ Reoxygenation Injuries. Front Mol Biosci 2021;8:698975. 